\title{
El papel de la familia \\ en la educación sexual
}

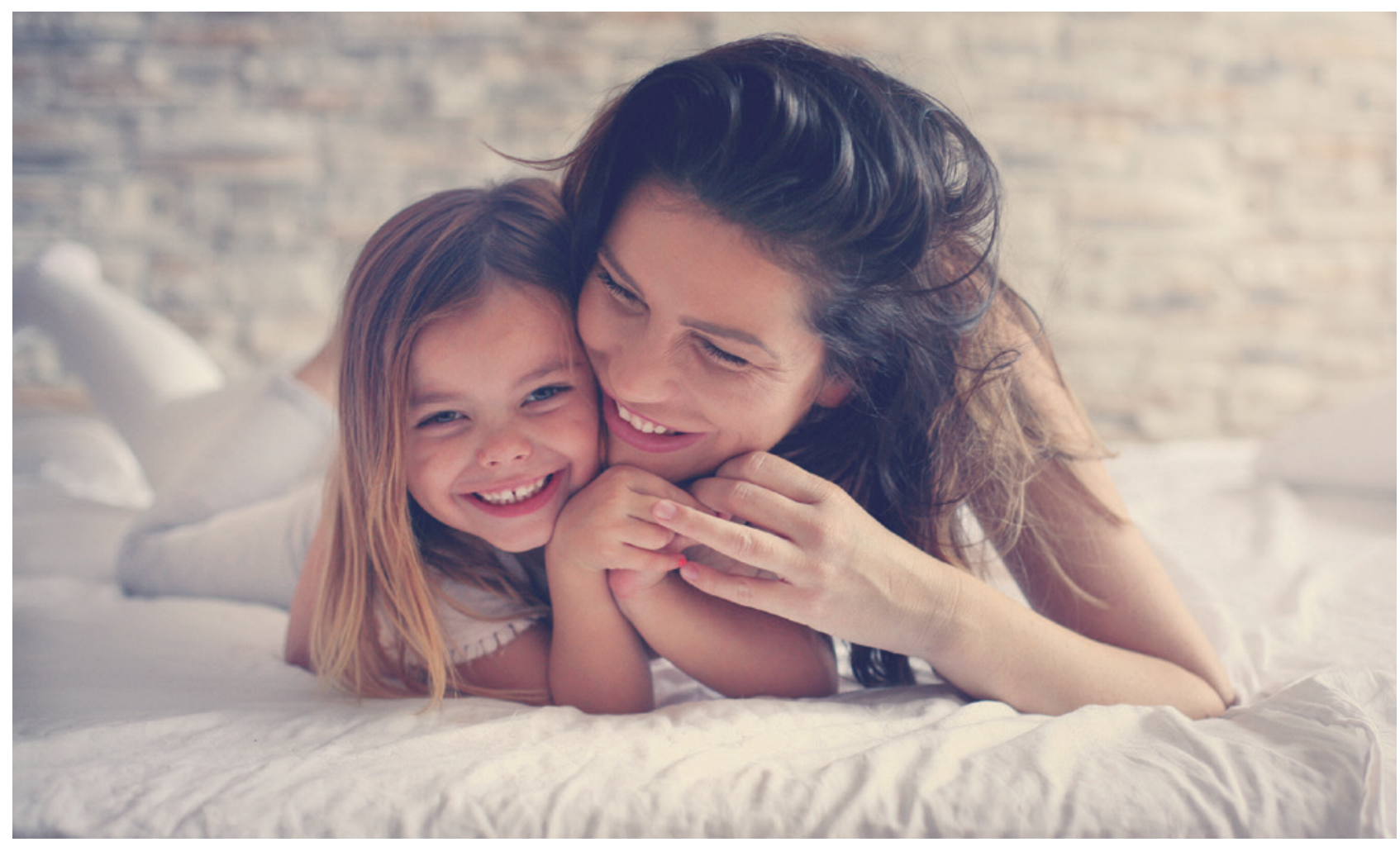

La educación sexual incluye la aceptación del cuerpo, la necesidad de emplearlo para la relación con otros y el descubrimiento de que buena parte de nuestra realidad individual, social y cultural es sexuada. Sexualidad es un

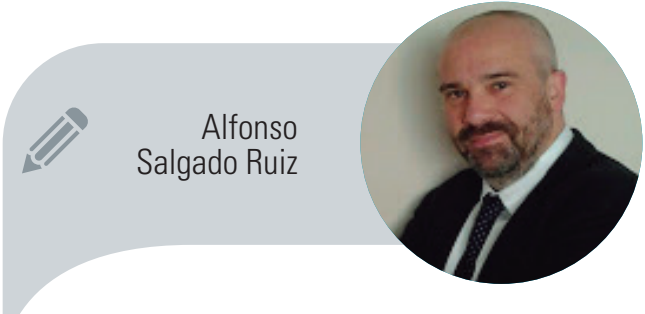

Facultad de Psicología. Universidad Pontificia de Salamanca asalgadoru@upsa.es término amplio y vivencial que debe tener siempre resonancias alegres, de crecimiento. La familia es la experiencia amorosa más genuina y cotidiana, y gracias al apego y la posibilidad de modelado es el entorno privilegiado para la educación sexual de una manera práctica. 
La base de la familia es el apego y su desempeño es la vida cotidiana. Los objetivos de la educación sexual se centran en la aceptación, la libertad y la salud. De este binomio familia-educación sexual se derivan consecuencias prácticas de enorme interés para docentes, consejeros, educadores, monitores, terapeutas.... porque la familia se debe considerar desde lo que constituye su elemento diferenciador, y es desde ahí como puede desarrollar su mejor cometido, que en ocasiones es insustituible. Y a la educación sexual se le debe asignar los objetivos y contenidos que realmente tiene, desde una perspectiva científica y amplia que le permita convertirse en una herramienta de crecimiento y felicidad.

Educar la sexualidad comprende hablar y practicar acerca del cuerpo, de su belleza, sus cambios y sus posibilidades, de los sentidos y su función necesaria para el conocimiento y la relación, supone abordar -en la teoría y desde la observación y la práctica- las relaciones con otros, las relaciones de pareja, el trato entre dos personas que se quieren, la expresión de afectos y emociones, el amor. También es educación sexual contribuir a que los hijos se sientan aceptados incondicionalmente y queridos, y por tanto aprendan a valorarse y quererse, tengan claro que como personas merecen la pena aunque siempre haya deseo de mejorar, y desde ahí, que busquen amistades y parejas que los cuiden, y sepan decir que no a lo que no desean. Y claro, educación sexual es también tratar acerca de genitales, de reproducción, de concepción y anticoncepción, de prevención, de placer, sensibilidades y emociones.

\section{Educar la sexualidad y vivir en familia: dos maneras de decir casi lo mismo}

Para abordar todos estos objetivos, cada uno de los cuales es notoriamente importante, no basta una sola herramienta, por muy necesaria que sea. Siguiendo ese famoso proverbio, si para educar a un niño hace falta toda una tribu, para la educación sexual entendida en este sen-

\section{Objetivos de la educación sexual}

ע Adquirir conocimientos adecuados y ciertos a su edad.

y Aceptar la sexualidad como una dimensión positiva.

y Procurar el desarrollo óptimo de la sexualidad.

y Valorar el propio cuerpo y desarrollar una adecuada autoestima.

$\checkmark$ Favorecer que hijos, padres y educadores puedan hablar con libertad y naturalidad.

y Favorecer la conciencia de que las conductas sexuales sean éticas.

y Adoptar roles de género flexibles y no discriminatorios.

\ Adquirir conciencia crítica sobre el uso actual de la sexualidad.

tido amplio derivado de lo que en la actualidad conocemos sobre la sexualidad, no basta sólo el colegio, ni sólo la familia, ni sólo otros recursos. Todavía más cuando aceptamos el reto de intentar hacerlo bien, es decir, entendiendo que la educación sexual no corresponde sólo a una etapa de la vida y que, aun centrándonos en la edad escolar, los objetivos son relativamente diferentes cuando se trata de niños pequeños, chavales un poco más mayores o adolescentes. En este sentido, y para centrarnos en el título que describe este trabajo, nuestra reflexión se centrará en el papel de la familia desde sus particulares funciones y desempeño.

La familia está constituida por personas que se quieren y se relacionan de una manera diferente al resto de los grupos sociales. $\mathrm{O}$ al menos, en parte. Así entendida, la familia como un sistema de relaciones de parentesco verticales y horizontales, es importante reconocer que el núcleo que la sustenta es la alianza o compromiso entre los cónyuges, normalmente en el contexto de cierto grado de intimidad entre los esposos, el vínculo de apego de los hijos con los padres (a veces, enriquecido también con el vínculo con los abuelos y otros familiares), los vínculos de cuidado paternomaterno filiales y el vínculo fraternal. Esto permite que cada hijo encuentre, por un lado, los cuidados y la atención propia del apego pero también, el modelado y la relación entre iguales con los hermanos y la observación de la relación cotidiana entre su padre y su madre. Todo un compendio de herramientas con las que aprender buena parte de los prerrequisitos de la educación sexual amplia y los propios contenidos y habilidad de esa misma educación sexual. Y hacerlo en el contexto particular que supone la familia como una 


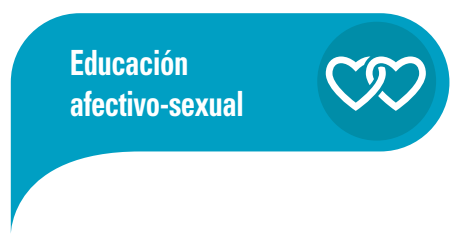

\section{Funciones de la familia}

y Ofrecer una relación afectiva tierna y cálida.

У Satisfacer las necesidades básicas biológicas, cognitivas, afectivas y sociales.

ע Aceptar a la persona tal como es, de forma incondicional.

y Ser fuente de información y transmisión de valores.

y Enseñar y obligar a sus miembros a comportarse de forma socialmente deseable.

ע Ayudar a buscar soluciones ante los problemas.

y Ofrecer modelos de imitación e identificación.

У Fomentar la autonomía en lugar de la sobreprotección.

y Exigir responsabilidades conforme a las capacidades de cada uno.

\ Ser núcleo social que sirve de descanso para recuperarse de esfuerzos y tensiones.

У Constituirse como el espacio más seguro y predecible.

y Ser espacio de participación activa, en la medida de la edad y capacidad de cada uno experiencia amorosa cotidiana, de aceptación de cada uno como es y de mutuo conocimiento, la familia como entidad protectora de cada miembro y la familia como principal colaboradora con otros elementos de educación y desarrollo, como la escuela (LóPEz, 2005). Parecería que las funciones de la familia coinciden en buena parte con los objetivos de la educación sexual, y sobre todo, con una metodología en la que no prima "hablar de" sino "practicar sobre".

Por eso, la familia constituye el núcleo más adecuado para el desarrollo de los niños y es vital en el desarrollo y autonomía de los adolescentes. Desde la perspectiva de la infancia, el núcleo de una familia lo forman unos adultos con capacidad para cuidar a un menor y un menor que se vincula a esos adultos.

El vínculo del apego responde a una de las necesidades humanas fundamentales, que es la necesidad de sentirse seguro, protegido por una o varias personas que sabemos incondicionales, disponibles y eficaces, y es un requisito para un desarrollo afectivo, social y sexual correcto, saludable y positivo. Cuando se habla de necesidades fundamentales, se quiere decir primarias, no aprendidas, con una base biológica propia de la especie a la que pertenecemos y, por tanto, con una clara función adaptativa. La tendencia a la unión, intimidad y placer en las relaciones interpersonales corresponde a tres necesidades básicas:
7 La necesidad de establecer vínculos afectivos percibidos como incondicionales y duraderos, esto es, la necesidad de apego.

$\boldsymbol{\lambda}$ La necesidad de disponer de una red de relaciones sociales (amigos, conocidos...), esto es, la necesidad de pertenencia.

$\boldsymbol{\lambda}$ La necesidad de contacto físico placentero, esto es, la necesidad de actividad sexual asociada al deseo, la atracción y el enamoramiento.

Cubrir estas tres necesidades supone asegurar la propia aceptación y el deseo de exploración de posibilidades, incluidas las que conducen al cambio de comportamiento, la pertenencia al grupo que implica una sociabilidad correcta y, por supuesto, la reproducción y la relación íntima y particular con otra persona. La insatisfacción de estas necesidades implica aumentar en gran medida la probabilidad de soledad emocional, soledad social y frustración sexual. En resumen, la naturaleza tiene previstos sentimientos de bienestar y gozo cuando tales necesidades se cubren, de infelicidad, desadaptación y sufrimiento en caso contrario.

La importancia del apego es crucial pues influye de manera decisiva en la formación de la personalidad y el desenvolvimiento social y sexual de la persona, ya que constituye patrones de conducta relativamente estables que mediatizan todas las relaciones sociales y muy especialmente las relaciones íntimas (LóPEz, 2008). Las figuras de apego y una adecuada vinculación a las mismas (estable, segura, accesible, cálida, exclusiva), ayudan al niño a construir un modelo de sí mismo y del mundo a partir del cual actúa, comprende la realidad, anticipa el futuro y construye sus planes. $Y$ es que esta relación tan especial, que fortalece la seguridad de los niños en sí mismos y en las relaciones con sus padres, permite aprender dos cosas:

$\boldsymbol{\lambda}$ La conflanza en los demás, lo que facilita las relaciones íntimas y sociales.

$\boldsymbol{\lambda}$ Y el lenguaje propio de las relaciones íntimas: mirar y ser mirados, acariciar 


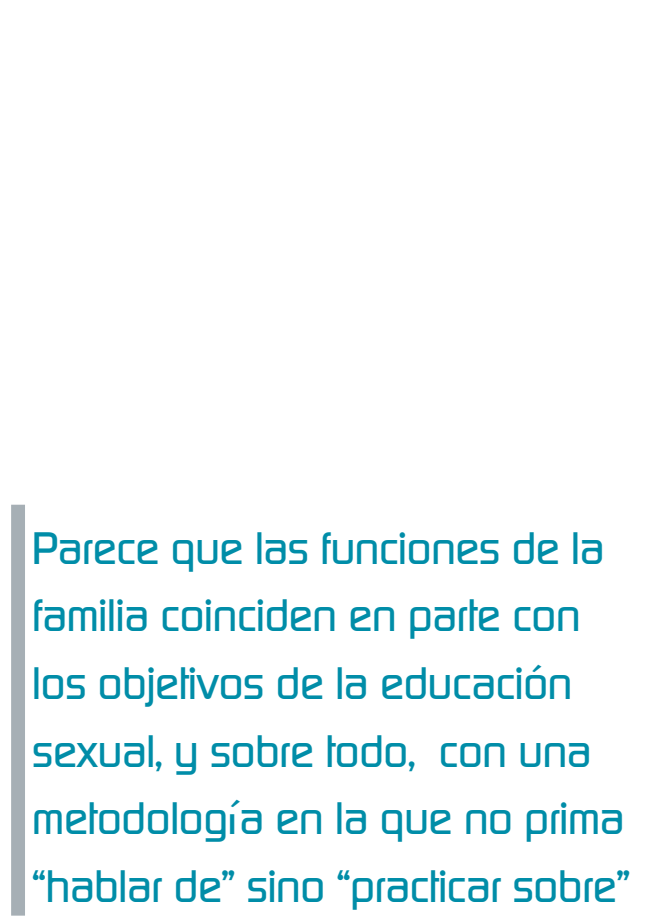

y ser acariciados, tocar y ser tocados, besar y ser besados, expresar admiración y ser admirados...

Por último, se ha de tener en cuenta el marco temporal en el que van poniéndose en funcionamiento todos estos elementos. Y es que el papel de la familia se juega en la vida ordinaria, en la rutina cotidiana, donde los mecanismos de aprendizaje operan de forma frecuente. Es la observación cotidiana del modo de comportarse la que actúa como elemento principal de modelado. Es también el proceso diario de reforzamiento de un determinado patrón de comportamiento y la extinción o castigo de otros, lo que termina moldeando el comportamiento. Por eso, no importa tanto la intensidad cuanto la frecuencia. $Y$ es que un niño que escucha comentarios positivos sobre su conducta o sus sencillos logros de forma contingente y frecuente termina aceptando que es una persona válida; un niño que observa a su padre y madre comportándose de forma similar, acaba flexibilizando los roles de género y entendiendo a los demás más como individuos que en función de su sexo; un niño que recibe caricias, besos, abrazos y que ve que sus hermanos también los reciben e incluso observa expresiones cariñosas de afecto entre sus padres, aprende a entender el cuerpo como mensajero y a valorar la ternura y el placer; un niño con quien se habla de todos los temas, intentando que se entiendan las palabras en función de su edad y capacidades, aprende que no debe haber temas ocultados por ser sucios o malos. Los padres son modelo de comportamiento siempre, lo hagan bien

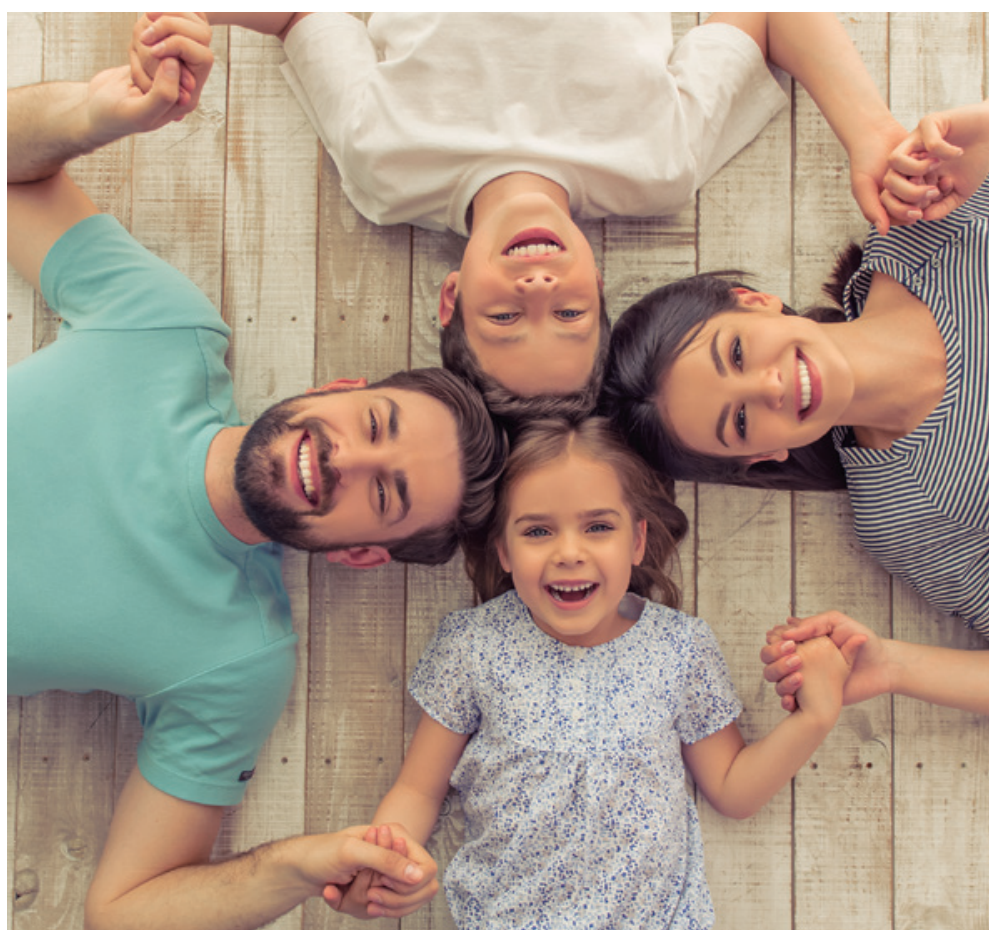

o lo hagan mal, pongan o no empeño en serlo. El acierto está en saber aprovechar esa oportunidad privilegiada.

\section{Total, que dos objetivos claros y cotidianos de la educación sexual en la familia}

Concretando, si los objetivos de la educación sexual se centran en conocerse, aceptarse y aprender a comportarse de forma sexualmente saludable (i.e. desde los roles sexuales flexibles hasta la correcta expresión de la erótica) y el papel que se le otorga a la familia en este campo viene determinado por la particular relación que se da en casa y lo frecuente del modelo de comportamiento, podríamos concluir que a los padres les toca transmitir dos cosas:

$\boldsymbol{7}$ Es posible quererse, tener pareja, entenderse, disfrutar a pesar de que no siempre todo es fácil... y que salga bien. Incluso, en el caso de padres separados es posible transmitir que hay experiencias de relación íntima y exclusiva que son fuente inmensa de alegría, de crecimiento y de felicidad, y que hay que saber cuidarlo en el día a día. Esto no se hace tanto conversando (que también) sino, sobre todo, pasando tiempo en familia y transmitiendo de forma cotidiana respeto, admiración, cariño, comprensión, ternura, atracción por la pareja. 


\section{(hii) CAMnInAกDO JUกTOS}

Para comentar en pareja mientras tomamos un café o paseamos tranquilamente: ¿Nos divertimos juntos? ¿Qué es lo que más me gusta hacer contigo? Y ya puestos, pongamos tres palabras (un color, un sabor y un sonido) a tus abrazos, tus caricias y tus piropos.

Oye ¿qué es lo que más me cuesta tratar con los chicos en esto de la educación sexual? ¿Qué recuerdas de tu experiencia con tus padres que sería bueno retomar o cambiar del todo? los vínculos afectivos y la intimidad, que incluyen la expresión afectiva y el compromiso; (4) de unas relaciones igualitarias entre hombre y mujer, pero también entre personas con independencia de su sexo, lo que incluye un trato personal a cada hijo en función de sus características personales y no por ser chico o chica; y (5) de la correcta expresión de los afectos, lo que implica saber reconocer las propias emociones y ser capaz de explicarlas, tener cierta dosis de autocontrol emocional y aprender (y practicar) a intercambiar afectos de forma mutua y respetuosa.

Siguiendo a López (2005), la función de los padres en la educación sexual se concreta en los siguientes objetivos:

त En primer lugar, ser adecuadas figuras de apego, desde el comienzo de la vida de sus hijos y a lo largo de la misma. Uno podrá desvincularse de su pareja, pero no debe caber la posibilidad de desvincularse de los hijos. Cambiará la forma y manifestación del apego en función de la edad de los hijos, pero siempre deberá caracterizarse por asegurar confianza, estima y afecto de forma incondicional y eficaz. Y en ese sentido, desde el principio debe vivirse la relación con los hijos desde un código de intimidad, que incluirá caricias, besos, abrazos y cuantas expresiones de cariño y ternura sean posibles, pero también los momentos de confidencialidad, de compartir sueños y proyectos, de respeto por sus opiniones y secretos, de intercambio de narración de vivencias siempre que haya deseo y libertad para hacerlo.

入 Ser modelos aceptables: (1) de aceptación de uno mismo a pesar de que haya cosas que se quieran mejorar (y en este sentido, ser ejemplo de persona capaz de reconocer sus fallos y sus errores y pedir disculpas por ello); (2) de una concepción positiva del ser humano, que incluye la aceptación de las diferencias, el respeto, una visión comunitaria y corresponsable de la persona, la genuina libertad responsable...; (3) del valor de
7 Responder a las preguntas que los hijos puedan hacer y, a veces, adelantarse a la pregunta que hay detrás de la pregunta (por ejemplo: un hijo puede preguntar si ser homosexual es una enfermedad porque desea tener información o porque desea saber la opinión de sus padres acerca de la homosexualidad, si es que duda acerca de su orientación sexual). Es decir, quizás convenga adelantarse a los sucesos evolutivos más importantes y anticipar sus intereses, dudas y motivaciones. En todos estos casos, hay que ser responsable y honesto y aportar siempre información legitimada, aunque no necesariamente tenga que coincidir con nuestras opiniones y valores.

Con los más pequeños, no censurar la sexualidad infantil saludable (e.g. exploración de su cuerpo incluyendo los genitales, jugar a que son personas del otro sexo, interesarse por el cuerpo desnudo de otras personas, imitar a los adultos besándose con otros niños...) y con los más mayores, hablar abiertamente de ciertos riesgos derivados de algunas prácticas sexuales, así como ofrecer ayudas.

入 Proteger de riesgos reales e imaginarios, que pueden ir desde los abusos sexuales hasta las distintas formas de violencia de pareja en adolescentes, desde las coacciones explícitas o sutiles para mantener cualquier tipo de relación sexual hasta la presión social y de los medios acerca de la imagen 


\section{Educación sexual desde la familia}

corporal que puede atentar contra la autoestima y puede ser un factor de riesgo para los trastornos de la conducta alimentaria, desde el acoso y la exclusión por tener que hacer obligatoriamente lo que hasta hace poco estaba mal visto o estaba prohibido, hasta el miedo a no ser aceptado en casa por ser homosexual o ser agredido por terceros por esa misma razón...

入 Colaborar con el colegio o el instituto en las actividades de educación sexual que se programen. En algunos casos, esas actividades incluyen la invitación a los padres; en otras ocasiones, son actividades para los propios padres y madres que organiza el AMPA, o la dirección del centro o alguna asociación educativa o sanitaria; y en muchas ocasiones serán actividades organizadas para los estudiantes, en las que siempre podremos mostrar interés (no más ni diferente del que mostremos por otras) y poder comentar en casa qué es lo que se está haciendo.

خ Y, por supuesto, saber buscar ayuda e información cuando no se sepa actuar o se tengan dudas acerca de hacerlo de forma correcta en algún asunto concreto.

Y antes que nada, y sobre todo, enseñarles y hacerles experimentar que existe la igualdad y el amor, y que ese amor se expresa en las relaciones entre los miembros de la familia, aunque no seamos una familia de película (porque no existen, ni siquiera en el cine), en la incondicional aceptación de cada hijo sea como sea, incluida su identidad sexual y la orientación

\section{parr SABEr mâs}

López, F. (2005). La educación sexual de los hijos. Madrid: Pirámide.

López, F. (2015). Ética de las relaciones sexuales y amorosas. Madrid: Pirámide.

Salgado, A. (2015). Diversidad y unidad: estructura, funciones y fortalezas de la familia. Sal Terrae, 103, 471-484. y Hacemos educación sexual continuamente, aunque no seamos conscientes: educamos a nuestros hijos a través de nuestras palabras y silencios, de nuestras conductas y de la expresión de nuestros afectos.

y Una educación sexual de calidad se orienta a que nuestros hijos aprendan a conocerse, aceptarse y a expresar su sexualidad de modo sano, adaptativo y feliz. La sexualidad es mucho más que genitalidad, mucho más que reproducción y mucho más que placer.

У Aprovechemos las oportunidades: una conversación, un programa en la tele..., pero también aprovechemos las ocasiones cotidianas de expresar emociones, dar mensajes de autoestima, valorar su cuerpo, darnos caricias, besos y abrazos, tratarnos como personas no como miembros de uno u otro sexo...

y Hay que estar dispuestos para dialogar. El diálogo no puede ser impuesto y conviene ser conscientes de la pregunta que hay "detrás de la pregunta". Y en todos los casos, tres consejos muy útiles: escucha, escucha y escucha.

У ¡Ah! Y cuando se trata de hablar de todo esto, recuerda que es normal que padres e hijos nos sintamos incómodos cuando tratamos estos temas en casa.

y No veamos con ojos de adultos lo que hacen los niños: seguramente caeremos en interpretaciones precipitadas.

У Respetar el pudor, los secretos y los silencios de nuestros hijos.

У Reforzar la capacidad que tienen nuestros hijos más mayores para tomar decisiones. Hacerles ver que nosotros estamos convencidos de ello. Cualquier momento es bueno para destacar que toda decisión debe ser personal. Depositar en ellos la responsabilidad propia para su edad y hacerles ver que las decisiones traen consecuencias y, por tanto, debe existir un mínimo de reflexión a la hora de decidir.

y Y por encima de todo, transmitamos de palabra, acción y gesto que la sexualidad, en todas sus dimensiones, es algo positivo, limpio, que nos hace crecer, disfrutar y ser felices, que pasa por el cuerpo pero también por las emociones, la razón y la relación social libre, igualitaria y respetuosa.

de su deseo, y en ofrecer un modo positivo de interpretar el mundo, la familia, la amistad y la relación de pareja. Y que nuestro amor incondicional hacia ellos se traduce también, cómo no, en la confianza que depositamos en otros (como sus profesores y el resto de educadores) para ayudarnos a hacerles crecer • HEMOS HABLADO DE

Educación sexual; familia; prevención.

Este artículo fue solicitado por PADRES Y MAESTROS en mayo de 2017, revisado y aceptado en octubre de 2017. 